\title{
An Improved Triggering Updating Method of Interest Message with Adaptive Threshold Determination for Directed Diffusion Routing Protocol
}

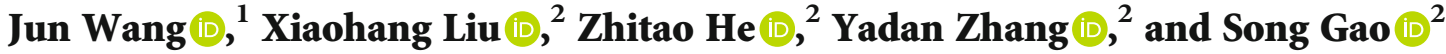 \\ ${ }^{1}$ School of Electrical Engineering, Henan University of Science and Technology, Luoyang, Henan 471000, China \\ ${ }^{2}$ School of Agricultural Equipment Engineering, Henan University of Science and Technology, Luoyang, Henan 471003, China \\ Correspondence should be addressed to Jun Wang; wangjun_haust@163.com
}

Received 30 September 2020; Revised 21 December 2020; Accepted 16 January 2021; Published 25 January 2021

Academic Editor: Mario E. Rivero-Angeles

Copyright (c) 2021 Jun Wang et al. This is an open access article distributed under the Creative Commons Attribution License, which permits unrestricted use, distribution, and reproduction in any medium, provided the original work is properly cited.

\begin{abstract}
Although some progress has been made in studying the triggering updating methods of interest message for reducing node energy consumption in directed diffusion routing protocols, they do not consider the anisotropy of sensing areas of nodes and the requirement of diversity of interest message exchange rates within real-time performance, which makes these studies unable to accurately adapt to the characteristics of interest message update. In this work, we proposed an improved triggering updating method (ITUM) consisted of adaptive threshold determination based on the analytic hierarchy process and update judgement by similarity comparison of interest messages. Meanwhile, the network model and the sensing model are presented to describe actual network scenarios. We analyze the impact of critical parameters and evaluate the performance of ITUM with several interest message updating methods from the aspect of lowering the number of information exchanges. The simulation results prove that ITUM can improve the adaptability to scene changes while decreasing the number of information exchanges compared with the existing methods. Furthermore, it is shown that ITUM is a highly effective solution for determining the triggering updating conditions of various information exchange rates in directed diffusion routing protocol.
\end{abstract}

\section{Introduction}

Query routing technique is undoubtedly one of the most typical routing protocols for wireless sensor networks (WSNs) [1], which is concretely composed by interest message diffusion, data transmission, and path enhancement in the implementation process [2-5]. The routing method has several remarkable highlights in data-oriented monitoring applications, such as direct transmission of interested data, information screening [6], and local communication [7], resulting in the advantages of less redundant information and long network lifetime. Moreover, the query routing protocol can be explicitly classified into rumor routing, directed diffusion routing, disjoint multipath routing, and braided multipath routing [8-11]. Directed diffusion routing exhibits excellent energy efficiency compared with other query routing methods [12] and can effectively lessen network data stream by query-driven data transmission and local data aggregation
[13]. For example, Intanagonwiwat et al. explored the use of directed diffusion for a simple remote-surveillance sensor network and enabled diffusion to achieve energy savings by selecting empirically good paths and by caching and processing data in-network [14]. Mu et al. inquired a directional diffusion routing protocol to improve the reliability of data transmission and extend the network life in wireless body area networks (WBANs) [15]. Directed diffusion routing is made up of three independent stages: interest diffusion, data transmission, and path enhancement [16-19]. During the application of directed diffusion routing, the query request is sent to nodes in the target area in the form of interest message, and the routing path is established in turn. Afterwards, the nodes start to execute the corresponding monitoring tasks according to the description of the interest message. Among them, the spread of interest message is named as interest message diffusion. Besides, since the network is not static, the nodes in the target area may move out, and on 
the contrary, the nodes outside the target area can also move in. These mobile nodes unavoidably require to communicate with surrounding nodes for timely exchanging or achieving interest messages in this phase, which is defined as interest message update.

In order to diminish energy consumption and extend network lifetime, various studies have been performed to optimize directed diffusion routing. For instance, Eghbali and Dehghan addressed a load-balancing method based on multipath directed diffusion to prolong the lifetime of nodes by establishing a multihop path between receivers and sensor nodes [20]. Li and Shi enhanced energy utilization rate and extended network lifetime by optimizing data transmission path and data aggregation [21]. Liu et al. investigated DDBC, a clustering-based directed diffusion routing protocol, to decrease network topology and hence improve energy efficiency by clustering and suppressing redundant messages in flooding [22]. However, these developed protocols solely pay attention to explore energy conservation strategies during data transmission and path enhancement stages in terms of data fusion, load balancing, and optimization path, less considering the energy consumption generated by interest message update. Nevertheless, the number of interest message exchanges in the stage of interest message update likewise directly influences node energy consumption [23-25], which is tightly associated with triggering updating method of interest message. It is necessary to take into account the message exchange requirement and communication cost of the target area for a triggering updating method.

Some triggering updating methods have been reported to improve the routing performance in interest message update. The current triggering updating methods can be separated into two categories: conventional triggering updating method (TUM) and energy-saving triggering updating method. The first method is that the mobile node accomplishes update through periodically broadcasting the update request of interest message to the neighbor nodes during its movement [26]. This behavior based on periodic triggering may substantially increase the number of interest message exchanges and cannot adapt to the movement path of mobile node due to the blindness of triggering. Meanwhile, the possible simultaneous responses of neighbor nodes are probable to induce information redundancy and network congestion. For overcoming the weaknesses of the classic triggering updating method, a series of energy-saving triggering updating methods are probed by refining the trigger and response patterns. Yu and Zhang and Cui and Cao investigated a method to effectively decrease the frequency of interest message updates by spreading interest messages only between cluster heads [27, 28]. Krishnamachari and Ahn proposed an extending ring query approach that can implement adaptive adjustment of query radius [29]. Niu et al. recommended an interest message updating method based on update triggering, delayed response, and incremental update to decrease update frequency [30]. Although the propagation between cluster heads or the limitation of update request range can restrain the surge of update frequency due to the flooding mode, the problem that updating operation and movement path are difficult to adapt to has not been solved, and there is an increased possi- bility of premature death of cluster heads. Moreover, these studies are all based on the ideal node model and do not consider the variations in sensing scope for different directions. Additionally, compared with the other methods, the incremental update proposed by Niu et al. can decrease update number while multiple nodes are within the sensing area of mobile node during the update phase. However, it will inevitably boost the amount of response inhibition messages transmitted and cannot prevent nodes with high proximity of interest message content from acting void updates.

The ideal sensing area of node is approximately circular. Though in the actual scene, the path attenuation of signal transmission in different directions is varied as a consequence of the influence of wireless device properties and other environmental factors such as obstacles, leading to the anisotropy of sensing area. On the other side, choosing the improper threshold based on previous experience is a crucial problem to the triggering updating method. This may cause a direct impact on the real time, adaptability, and timeliness of interest information update. It is additionally a vital concern to avoid updating between a mobile node and multiple fixed nodes with high-similarity message content.

Based on the above introduction, this paper proposes an improved triggering updating method for interest message update in directed diffusion routing protocol, which is constituted by adaptive threshold determination using the analytic hierarchy process (AHP) and trigger judgement adopting similarity comparison of interest messages. The contribution of this paper covers four aspects:

(1) We develop a network model based on different overlapping patterns of sensing areas between a mobile node and various fixed nodes and establish a sensing model considering time-varying anisotropy of wireless signal transmission

(2) We propose an improved triggering updating method comprised of threshold determination and update judgement to minimize the number of information exchanges under different information exchange rates

(3) We use the Gauss-Markov mobility model to describe the mobile node movement in the target area and investigate the performance of reducing the number of information exchanges for diverse network and mobility parameters

(4) Simulation results demonstrate that the proposed triggering updating method can significantly decrease the number of information exchanges due to the combination of advantages of two factors. The analytic hierarchy process can dynamically obtain the proper threshold of interest messages of multifixed nodes, and the similarity comparison is capable of avoiding unnecessary updates

The outline of this paper is organized as follows. Section 2 provided the network model and sensing model. Section 3 
depicts the proposed method. In Section 4, we conduct the performance analysis of the proposed method under different network and mobility parameters. Finally, we summarize our work and draw conclusions in Section 5.

\section{Network and Sensing Model}

In this section, aiming to describe the scenario of interest message update precisely, we propose several assumptions of network model and two categories of overlapping pattern of sensing areas to imply the preconditions of update triggering in consideration of the bidirectional links between nodes and use a time-varying standard normal distribution to indicate the difference of sensing area of node in each direction.

2.1. Network Model. Considering the distribution of fixed nodes, the moving path of mobile node, and the capacity of node interest messages, we concretely define network model as follows.

(1) Every node has the same communication ability, each communication link between nodes is bidirectional, and all fixed nodes in WSNs are randomly distributed in a rectangular target area

(2) The total number of nodes in the target area is $Q$, and each node can utilize the existing wireless location technology to provide location information

(3) The number of interest messages carried within each sensor node is $L$. These interest messages vary and are sorted by importance in descending order. The characters $1,2, \ldots, K$ are used to depict different monitoring tasks, respectively. Additionally, it is assumed that $K$ is greater than L. Meanwhile, the interest message in a mobile node is empty at the initial stage

(4) The overlapped sensing areas of fixed nodes manifest that there are common interest messages (monitoring tasks) between them and vice versa. The fixed nodes overlapping with a mobile node can be abstracted as a fixed node

(5) A mobile node can enter or leave from any boundary of the target area and move according to the Gauss-Markov mobility model. The update is triggered by simultaneously satisfying the two conditions that the sensing areas of the mobile node and fixed nodes overlap, and the similarity of interest messages is higher than threshold value

(6) The overlaps can be divided into two kinds by the number of fixed nodes overlapped with a mobile node, as illustrated in Figure 1. The first category is that the sensing area of mobile node overlaps with that of a single fixed node, and triggering updating is settled by both. The second category is that the sensing area of mobile node overlaps with that of multiple fixed nodes at the same time, which can be classified into the following three cases:

(a) The sensing areas of fixed nodes are entirely overlapped, that is, the interest messages are the same, and the triggering updating is decided by the mobile node and any node together

(b) The sensing areas of fixed nodes do not overlap with each other at all. Namely, the interest messages are entirely different, and the mobile node needs to make the judgment of triggering updating with each fixed node, respectively

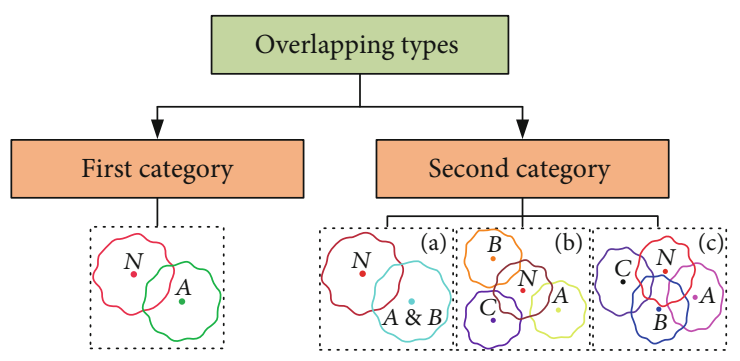

Figure 1: Overlapping patterns. $N$ represents the mobile node; $A, B$, and $C$ express fixed nodes, respectively.

(c) The sensing areas of fixed nodes are partially intersected, and the interest messages of these nodes are supposed to be not exactly the same. In this case, common messages of these fixed nodes are firstly arranged in random order, and the remaining messages are further sorted in descending order of importance of interest message content until the message capacity of a fixed node is reached. The triggering updating assessment is eventually carried out between the reorganized interest message and the interest message of mobile node

(7) The query request is strictly restricted in the target area, and the sink node locates outside the target area. Each request by the user is time-limited and expires after the corresponding validity duration

2.2. Sensing Model. Radio irregularity originated by the heterogeneous properties of devices and the anisotropic properties of propagation media is a common and nonnegligible phenomenon in WSNs [31]. It results in irregularity in radio range and variations in packet loss in different directions for sensor node. The sensing model we put forward here is inspired by three properties of radio signals: anisotropy, continuous variation, and heterogeneity. In addition, the degree of influence of factors such as residual energy of nodes and neighboring environment on the perceived distance of nodes in various directions is random, time varying, and has the characteristics of normal distribution. Therefore, for the convenience of description and application, the standard normal distribution is used to demonstrate the random influence degree of multiple independent factors on the perceived distance of nodes in each direction, and the time-varying process of the influence is expressed by introducing the time variable.

A time-varying irregular circle with the node as the center is proposed to depict the sensing area of node in the deployment scenario. The variable perceived distance $R_{s}\left(R_{s}\right.$ $\left.=\left(1-\sigma^{\prime}\right) R\right)$ is used as its radius, where $R$ denotes the radius as the sensing area of node is a regular circle $[32,33]$ and $\sigma$ $'\left(0<\sigma^{\prime}<0.2\right)$ is anisotropic influence factor.

The anisotropy influence factor $\sigma^{\prime}$ is represented by an integral function of the standard normal distribution with time and angle as variables and can be calculated by Eq. (1).

$$
\sigma^{\prime}=\int_{1 / 4|\sin (l \theta+t l)|}^{1 / 4|\sin (l \theta+t l)|} \frac{1}{\sqrt{2 \pi}} e^{-u^{2} / 2} d u
$$


where $\theta(\theta \in[0,2 \pi])$ and $u(u \in(-0.25,0.25))$ are direction coefficient and adjustment coefficient, $l$ represents a random number greater than $1, t(t>0)$ indicates time, and the product of $t$ and $l$ is an adjustable time-varying parameter.

\section{Triggering Updating Method}

The interest message update process has varied requirements on the degree of information exchange between a mobile node and several fixed nodes with overlapped sensing areas, corresponding to different information exchange rates. The high information exchange rate indicates that the update sensitivity of interest message of mobile node and fixed nodes is at a high level and vice versa. For the information exchange requirements, relying only on the previous experience to artificially set the update threshold, it will inevitably lead to the decline of necessity and adaptability of update. Therefore, we build an improved triggering updating method in this section. In this method, the update threshold of fixed nodes is solved adaptively based on AHP for evaluating the difference of interest message content in light of information exchange rate, and then the similarity of interest message contents between the mobile node and fixed nodes is computed and compared with the update threshold to judge whether to update or not.

3.1. Threshold Determination. AHP proposed by Saaty in the early 1970 s is a multicriteria decision method that uses hierarchical structures to represent a problem and makes decisions based on importance scales [34, 35]. Because of the effective combination of qualitative and quantitative approaches, it has been popularly practiced in the fields of performance evaluation, engineering planning, program sequencing, economic management, etc. [36]. In this paper, the complex correlation and subordination between interest messages and information exchange rates are combined into a three-level analysis structure model by AHP, and the threshold determination can be converted into computing the relative weights of information exchange rates to the update threshold. The specific steps are listed as follows:

(1) Establishing Hierarchical Threshold Model. The information exchange rate and message priority are arranged into an ordered hierarchy model based on membership relations, as shown in Figure 2. The update threshold $z$ is referred to as the overall target layer, the priorities $\left(B_{1}, B_{2}, \cdots, B_{L}\right)$ of interest message content are employed as the criterion layer for achieving the overall goal, and three information exchange rates are selected as the solution layer in which $A_{1}, A_{2}, A_{3}$ express high, medium, and low information exchange rates, respectively. In the constructed model, the update threshold $z$ is jointly determined by priorities $B_{1}, B_{2}, \cdots, B_{L}$. A priority $B_{j}(1<j<L)$ is mutually affected by the three information exchange rates and the priorities in turn act on the same information exchange rate.

(2) Constructing Importance Matrix. According to the importance of priority levels of interest message content in the criterion layer, the criteria scale is evenly subdivided into nine levels from equal importance (lowest priority) to extreme importance (highest priority), and the values are assigned 1 to 9 . Each value signifies that the importance of the former over the latter of two priorities compared, and the corresponding value should be reciprocal with the positions of two priorities interchange. Moreover, $B_{j 1}, B_{j 2}, \cdots$, $B_{j L}$, the importance comparison results of $B_{j}(1<j<L)$ and $B_{1}, B_{2}, \cdots, B_{L}$ are added as the $j$ th row element of importance matrix of criterion layer $B$ using the update threshold $z$ as the evaluation criterion, and the importance matrix $B$ is set up as follows:

$$
B=\left[\begin{array}{ccc}
b_{11} & \cdots & b_{1 L} \\
\vdots & \ddots & \vdots \\
b_{L 1} & \cdots & b_{L L}
\end{array}\right]
$$

As a result of the identical hierarchical model, the importance matrix of solution layer and criterion layer is assembled by the same method. Through the steps above, the importance matrix of solution layer with priority $B_{j}(1<j<L)$ as evaluation criteria is described by Eq. (3).

$$
A_{B_{j}}=\left(\begin{array}{lll}
a_{11} & a_{12} & a_{13} \\
a_{21} & a_{22} & a_{23} \\
a_{31} & a_{32} & a_{33}
\end{array}\right) .
$$

(3) Sorting and Validation of Single-Layer Weight. The eigenvectors of importance matrices $B$ and $A_{B_{j}}$ can be obtained by the square root method. The relative weights $W^{B_{j}}$ among $A_{1}, A_{2}, A_{3}$ and the relative weights $W^{z}$ among $B_{1}, B_{2}, \cdots, B_{L}$ can be collected after normalization, respectively. In this paper, we use the calculation process of $W^{B_{j}}$ as an example, to illuminate the same implement process of the two weights as follows.

(a) Compute the cubic root of product of elements of each row in the importance matrix $A_{B_{j}}$ by $W_{i}=\left(\prod_{j=1}^{3} a_{i j}\right)^{1 / 3}$ ,$(i=1,2,3)$ and normalize $W_{i}$ by the following:

$$
W_{i}^{B_{j}}=\frac{W_{i}}{\sum_{i=1}^{3} W_{i}}
$$

where $W_{i}^{B_{j}}$ represents the weight of $A_{i}$ by the use of $B_{j}$ as evaluation criteria. $W^{B_{j}}=\left(W_{1}^{B_{j}}, W_{2}^{B_{j}}, W_{3}^{B_{j}}\right)^{T}$ is the relative weight, namely, the normalized vector, and each component is the weight value of different information exchange rates relative to the priority $B_{j}$.

(b) Calculate the largest characteristic root of importance matrix $A_{B_{j}}$ by Eq. (5).

$$
\lambda_{\max }=\sum_{i=1}^{3} \frac{C_{i}}{3 W_{i}^{B_{j}}},
$$

where $C_{i}$ is the $i$ th element of vector $C\left(C=A_{B_{j}} W^{B_{j}}\right)$. 


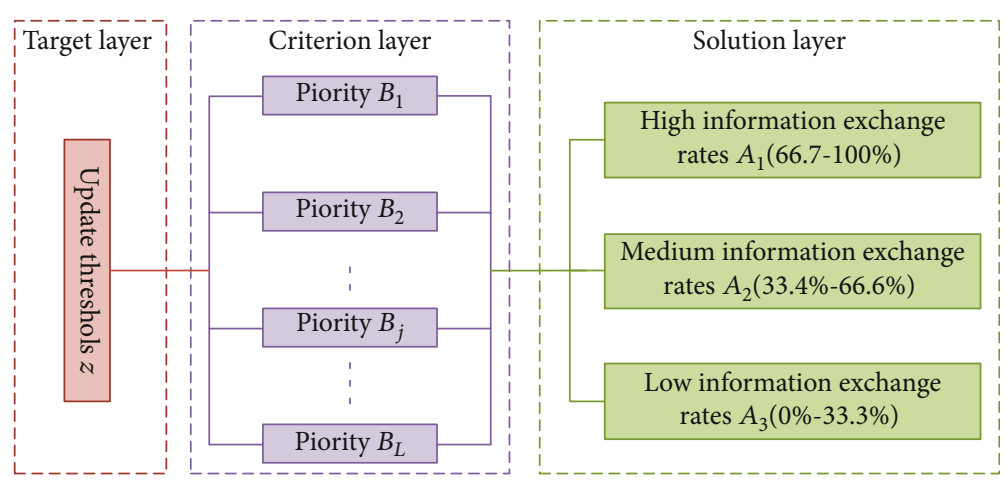

Figure 2: Hierarchical threshold model.

(c) As the elements of importance matrix are quantitatively assigned, in order to avoid the influence of change of characteristic roots caused by data contradiction on calculation result and decision accuracy, the consistency validation on its elements can be done through Eq. (6).

$$
\mathrm{CR}=\frac{\mathrm{CI}}{\mathrm{RI}}=\frac{\lambda_{\max }-n / n-1}{\mathrm{RI}}=\frac{\lambda_{\max }-3}{1.16}
$$

where CR is the consistency ratio, RI is the random consistency index, $\mathrm{CI}$ is the consistency index, and $n$ is the order of matrix $A_{B_{j}}$ with a value of 3 . CR is used to indicate the degree to which the constructed importance matrix deviates from the ideal completely consistent matrix, which can effectively detect the logical errors in judgement and improve the reliability of the final decision. The threshold value of 0.1 is recommend by Saaty and has been widely accepted as a general setting used to assess whether the matrix has satisfactory consistency. In case consistency ratio $\mathrm{CR}=\mathrm{CI} / \mathrm{RI}<0.1$, it shows that importance matrix $A_{B_{j}}$ has satisfactory consistency. The normalized eigenvector $W^{B_{j}}$ can be applied as the weight vector representing relative weight. Otherwise, the element $a_{i j}$ should be adjusted through the reconstruction of $A_{B_{j}}$ by the most inconsistent element (MICE) iteration algorithm, which is recommended by Yuan and Liang [37].

(d) Weight Sorting and Threshold Determination. Based on consistency validation, the total weight of each information exchange rate can be computed by Eq. (7).

$$
W_{A_{t}}^{Z}=\sum_{j=1}^{L} W_{i j}^{B_{j}} W_{j}^{Z}, i=1,2,3,
$$

where $W_{A_{i}}^{Z}$ is the total weight of $A_{i}$ relative to $z, W_{i j}^{B_{j}}$ is the weight of $A_{i}$ relative to $B_{i}$, and $W_{j}^{Z}$ denotes the weight of $B_{i}$ relative to $z$. To ensure the reliability of the total weights obtained, the consistency validation can be designed:

$$
\mathrm{CR}^{\prime}=\frac{\sum_{j=1}^{L} W_{j}^{Z} \mathrm{CI}_{j}^{\prime}}{\sum_{j=1}^{L} W_{j}^{Z} \mathrm{RI}_{j}^{\prime}},
$$

where $\mathrm{CR}^{\prime}$ is the final consistency ratio and $\mathrm{CI}_{j}^{\prime}$ and $\mathrm{RI}_{j}^{\prime}$ are the sorting consistency index and random consistency index of hierarchical weights of solution layer to the criterion layer $B_{j}$. In the case of $\mathrm{CR}^{\prime}<0.1$, it is concluded that the weight sorting has satisfactory consistency. Then, the optimal threshold $z^{\prime}$ at the current information exchange rate can be gained by Eq. (9) and Eq. (10).

$$
\begin{gathered}
z^{\prime}=z_{\min }+\left(z_{\max }-z_{\min }\right) \times\left(1-W_{A_{i}}^{z^{\prime}}\right), \\
2 \sqrt{\frac{2 \sum_{i=1}^{L / 2}(K-(2 i-1))^{2}}{L}(L \text { is even }),} \\
\sqrt{\frac{2 \sum_{i=0}^{(L-1) / 2}(K-(2 i+1))^{2}-(K-L)^{2}}{L}}, \\
\times\left(2-\sqrt{\frac{2(K-L) / K+1}{\sum_{i=0}^{(L-1) / 2}(K-(2 i+1))-(K-L)}(L \text { is odd })}\right),
\end{gathered}
$$

where $z_{\max }$ is the maximum threshold, $z_{\min }$ is the minimum threshold with the value of zero, and $W_{A_{i}}^{z^{\prime}}$ is the weight of the current information exchange rate relative to the update threshold $z$ achieved by Eq. (7).

3.2. Update Judgement. After obtaining the update threshold of fixed nodes overlapped with a mobile node, the similarity can be adopted to assess whether the interest messages of mobile and fixed nodes satisfy the update condition under the current information exchange rate. Distance measurement methods are widely used to signify the similarity between data. However, such methods often show more reliable measurement results in low-dimensional space and weak performance in high-dimensional space by the cause of the sparsity of data [38]. Hence, the application of the distance-based method to the similarity comparison of multidimensional interest messages will unavoidably lead to a sharp increase in update times with the growing number of deployed nodes, forming a dimensional disaster. The predicament of the distance-based method results in the ignorance of similarity difference for high-value data and low-value 
data in various dimensions, which directly generates the underestimate of similarity outcomes. To solve this problem, we propose a modified distance-based similarity function by using the absolute average of each dimension to balance the similarity discrepancy of high-value data and low-value data. This function evaluates the similarity of each dimension of data, and its value is between 0 and $z_{\max }$. The larger the value, the higher the difference between the two information messages. The similarity of interest message contents between a mobile node and multiple fixed nodes is defined by the following:

$$
D(M, N)=\sqrt{\frac{\sum_{i=1}^{L}\left(M_{i}-N_{i}\right)^{2}}{L}} \times\left(2-\frac{\left|\sum_{i=1}^{L}\left(M_{i}-N_{i}\right) / m_{i}\right|}{\sum_{i=1}^{L}\left|\left(M_{i}-N_{i}\right)\right|}\right),
$$

where $M$ represents a mobile node and $N$ is the fixed node abstracted by the fixed nodes overlapped with $M ; M=\left(M_{1}\right.$, $\left.M_{2}, \cdots, M_{j}, \cdots, M_{L}\right)$ and $N=\left(N_{1}, N_{2}, \cdots, N_{j}, \cdots, N_{L}\right)$, respectively, indicate the interest message contents of $M$ and $N$, $M_{i}, N_{i} \in(1, K) ; m_{i}$ is the absolute average of $M_{i}$ and $N_{i}$.

The introduction of parameter $m_{i}$ can make the similarity analysis based on distance depends not only on the difference between $M_{i}$ and $N_{i}$ but also on the size of the dimension data, for intensifying the similarity measurement performance of dimension with large value in the case of high dimension. For example, suppose $M=(1,4,7)$ and $N=(3.6 .9)$, if $m_{i}$ in $D(M, N)$ is removed, then the similarity of $M$ and $N$ in these three dimensions is 2 , respectively, and the overall similarity is 2 . However, based upon data comparison, the similarity of the second dimension should be higher than that of the first dimension, and the similarity of the third dimension should be higher than that of the second dimension. According to the $D(M, N)$, the similarity of the two messages in the three dimensions is $3,3.6$, and 3.75 , respectively, and the overall similarity is 3.45 . It is easy to find that the similarity comparison function proposed in this paper has high-grade discrimination performance.

We decide whether to do the update operation following the similarity calculation. If the similarity is higher than the update threshold, the update operation will be enabled. Otherwise, the update will be abandoned, and the mobile node continues to move and prepares for the next update as requested by the query.

\section{Results and Discussion}

In the simulations, we make use of Gauss-Markov mobility model to depict the mobile node movement in the target area, which was initially presented for the simulation of a personal communication service network. It can be described as

$$
\begin{aligned}
& v_{k}=\beta v_{k-1}+(1-\beta) v_{\mathrm{avg}}+\left(\sqrt{1-\beta^{2}}\right) w_{v_{k-1}}, \\
& d_{k}=\beta d_{k-1}+(1-\beta) d_{\mathrm{avg}}+\left(\sqrt{1-\beta^{2}}\right) w_{d_{k-1}},
\end{aligned}
$$

where $v_{k}$ and $d_{k}$ are the new velocity and direction of mobile node at time interval $k$; $v_{\text {avg }}$ and $d_{\text {avg }}$ are constants representing the mean value of velocity and direction as $k \rightarrow \infty ; \beta(0$ $\leq \beta \leq 1)$ is the tuning parameter used to vary the randomness; $w_{v_{k-1}}$ and $w_{d_{k-1}}$ are random variables from a Gauss distribution. At each time interval, the next location is calculated based on the current location, velocity, and direction of movement. Specifically, at time interval $k$, the location of mobile node is given by

$$
\begin{aligned}
& x_{k}=x_{k-1}+v_{k-1} \times \cos \left(d_{k-1}\right), \\
& y_{k}=y_{k-1}+v_{k-1} \times \sin \left(d_{k-1}\right),
\end{aligned}
$$

where $\left(x_{k}, y_{k}\right)$ and $\left(x_{k-1}, y_{k-1}\right)$ are the $x$ and $y$ coordinates of mobile node at the $k$ th and $(k-1)$ th time intervals, respectively, and $v_{k-1}$ and $d_{k-1}$ are the velocity and direction of mobile node, respectively, at the $(k-1)$ th time interval. We set the target area to be $200 \mathrm{~m} \times 100 \mathrm{~m}$. All the simulation results are the average values of 50 simulations. The simulation parameters are shown in Table 1.

With the change of simulation parameters, the ITUM proposed in this paper is compared with traditional updating method (TUM) and TURIR [30], by using the number of information exchanges as the indicator.

Figure 3 depicts the relations of ITUM, TUM, and TURIR between the number of information exchanges and the deployment density of fixed nodes $m$. It is easy to observe that the number of information exchanges can increase significantly with the growth of $m$. When $m \in\left[1 / 1000 \mathrm{~m}^{2}, 1 /\right.$ $200 \mathrm{~m}^{2}$ ), the number of information exchanges of these three methods will rise slowly, with average growth rates of $21.58 \%, 23.73 \%$, and $21.72 \%$, respectively. When $m \in(1 /$ $\left.200 \mathrm{~m}^{2}, 1 / 100 \mathrm{~m}^{2}\right)$, the number of information exchanges of these three methods will proliferate, with the growth rates of $101.72 \%, 104.56 \%$, and $162.81 \%$ separately. Apparently, TUM is the most sensitive to the deployment density of fixed nodes, and ITUM has the minimum increase in the number of information exchanges, with the $m$ rising from $1 / 1000 \mathrm{~m}^{2}$ to $1 / 100 \mathrm{~m}^{2}$. The triggering methods of three methods lead this phenomenon. The timing update mechanism of TUM is associated with time and deployment density of fixed nodes. Under a certain time, the deployment density directly impacts the number of information exchanges, and the higher the $m$ is, the faster the increase will be. TURIR merely updated while the node displacement is higher than the fixed threshold value. Compared to TUM, the method can efficiently decrease the number of information exchanges, and the number of information exchanges will slowly increase with the increment of $m$ affected by mobility model and threshold value. However, if the node density increases to a specific value, the node displacement becomes the chief influencing factor, which will drive to the sharp increase in the number of information exchanges. ITUM can lessen the number of information exchanges by avoiding blind and invalid updates as a consequence of the triggering updating conditions recommended in this paper. Influenced by the anisotropy, update threshold, and similarity in the initial 
TABLE 1: Simulation parameters.

\begin{tabular}{lccc}
\hline Parameter & Symbol & Initial value & Variation range \\
\hline Deployment density of fixed nodes & $m$ & $1 / 200 \mathrm{~m}^{2}$ & {$\left[1 / 1000 \mathrm{~m}^{2}, 1 / 100 \mathrm{~m}^{2}\right]$} \\
Sensing radius of nodes & $R$ & $10 \mathrm{~m}, 55 \mathrm{~m}]$ \\
Tuning parameter & $\beta$ & 0.3 & {$[10 \mathrm{~m}, 5,1]$} \\
Average velocity of mobile node & $v_{\text {avg }}$ & $1 \mathrm{~m} / \mathrm{s}$ & $(0,1.25 \mathrm{~m} / \mathrm{s})$ \\
Average direction of mobile node & $d_{\text {avg }}$ & $\pi / 2$ & $(0, \pi)$ \\
Dimension of interest message & $L$ & 1 & {$[1,9]$} \\
Information exchange rate & $H$ & $50 \%$ & $(0,100 \%)$ \\
\hline
\end{tabular}

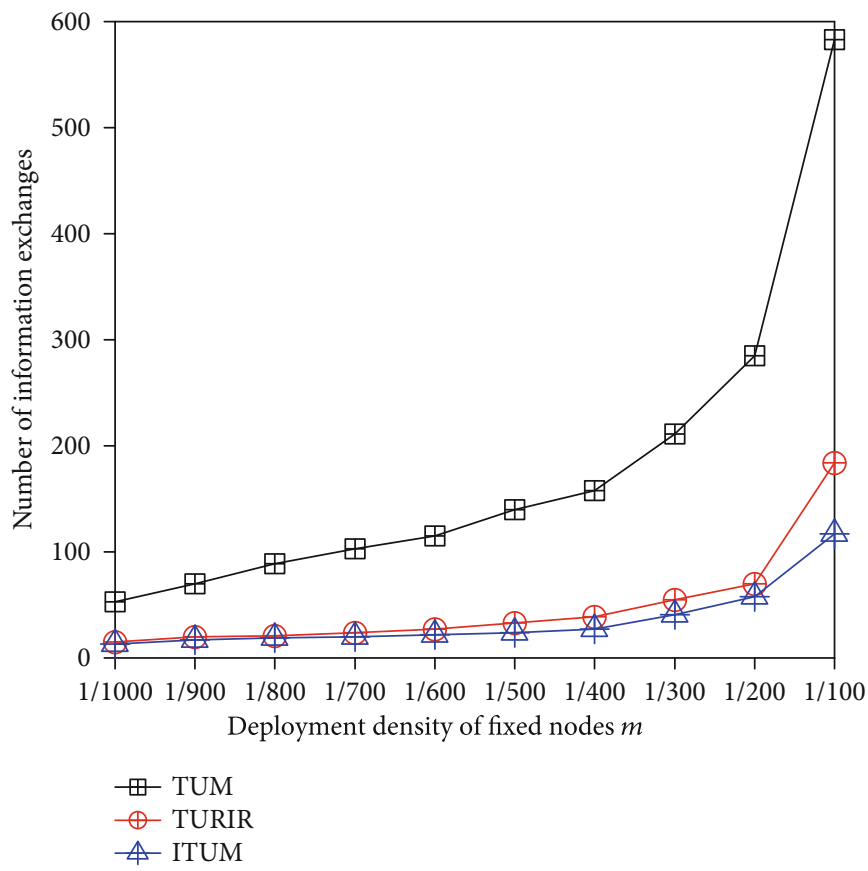

FIgURE 3: Number of information exchanges of three methods in different deployment densities.

phase, the number of information exchanges rises moderately with the increase of $m$. As the deployment density continues to grow, the anisotropy converts to the principle aspect and will cause an abrupt jump in the number of information exchanges.

As shown in Figure 4, with the increase of $R$, the average number of information exchanges of ITUM is lowered by $75.72 \%$ and $41.00 \%$, respectively, compared to that of TUM and TURIR. When $R \in[10 \mathrm{~m}, 30 \mathrm{~m})$, the number of information exchanges of ITUM and TURIR has an approximate trend with a slow growth rate. In particular, when $R \in[30 \mathrm{~m}$ , $55 \mathrm{~m}$ ], ITUM can dramatically decrease the number of information exchanges, compared with the other two kinds of methods. As a whole, the rate of change, the number of information exchanges, and the increment of ITUM are significantly lower than the other two methods produced by the anisotropy of sensing area and the triggering updating conditions, which diminishes the probability of the update judgment. Furthermore, it is easy to understand that the bigger the $R$ is, the more the number of information exchanges are, which means that more updates will be triggered. Therefore, in order to reduce the update times, designers should set reasonable sensing radius for network nodes.

Figure 5 demonstrates the relation between the dimension of interest message $L$ and the number of information exchanges. With the increase in $L$, the average number of information exchanges of these three methods is raised by $3.19 \%, 6.81 \%$, and $4.55 \%$, respectively. Compared with TUM, TURIR, and ITUM, it has better performance in reducing the number of information exchanges and decreases by $20.68 \%$ and $2.93 \%$ on average. When $L<4$, affected by the number of monitoring tasks, priority, and threshold range, TURIR is the most effective in cutting down the number of information exchanges by reason of the fixed threshold mechanism. When $4 \leq L \leq 7$, the number of information exchanges of ITUM can be stable at 38 , which has a prominent advantage over the other two methods. In the case of $L>7$, although the impact of $L$ will lead to sudden increases in quantities, contrasted to the other two methods, the number of information exchanges of ITUM is still 


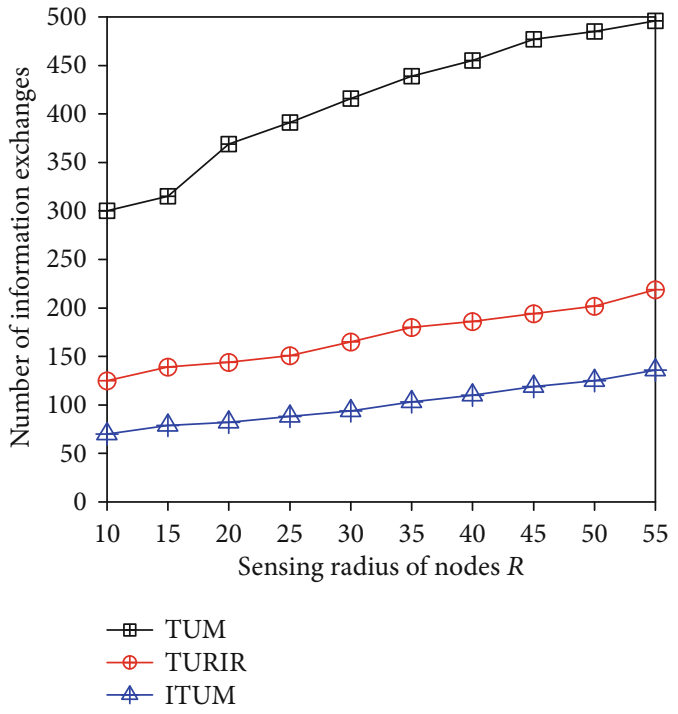

Figure 4: Relation between $R$ and number of information exchanges for different methods.

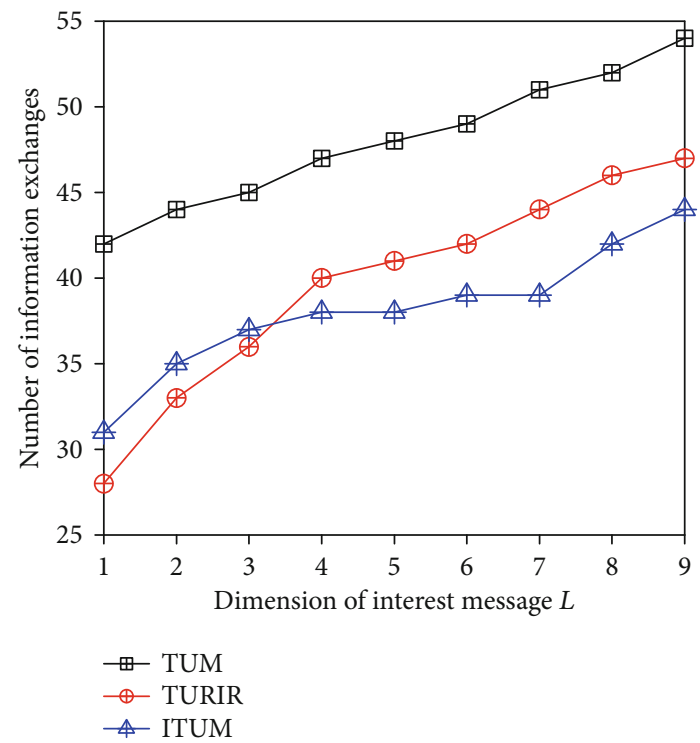

FIGURE 5: Influence of $L$ on number of information exchanges for three methods.

reduced by $20.38 \%$ and $8.76 \%$, respectively. Therefore, to lower the update times by ITUM, we need to limit the number of $L$ within a reasonable scale $(4 \leq L \leq 7)$.

As shown in Figure 6, with the increase in number of interest message update intervals from 1 minute to 20 minutes, ITUM is the least in the number of update messages in comparison with that of TUM and TURIR. The average number of update messages is reduced by $68.03 \%$ and $17.47 \%$, and the average amount of update data declined by $78.41 \%$ and $41.35 \%$, respectively. In Figure 6(a), the number of update messages in TUM is linked to the number of neighbor nodes, depending on the triggering way. In the condition of a definite deployment density of fixed nodes, the number of update messages decreases insignificantly with the increase of interest message interval, primarily induced by the decrease of the number of messages used for interest message propagation. The variance in the number of update messages between ITUM and TURIR is affected by the triggering updating mode, with the condition that the frequency of query request, the scope of target area, and the validity period of request remain unchanged.

Two main factors interlock with the amount of update data: whether to adopt incremental update and the number of messages in the update process, as exhibited in Figure 6(b), as TUM does not use the incremental update, the number of messages in the update process and message format bring about that its data quantity varies little and is much larger than the other two methods. In the case that ITUM and TURIR both utilize incremental update pattern, the amount of update data of both has direct relevance to the number of messages generated by respective triggering updating methods and message formats. In summary, ITUM can not only reduce the number of update messages in the update process but also match the update messages with user queries, which is particularly suitable for data-centric WSNs with low network bandwidth requirements.

Figure 7 shows the performances under various parameters of Gauss-Markov mobility model in terms of $d_{\mathrm{avg}}, v_{\mathrm{avg}}$, and $\beta$. With the growth of $d_{\text {avg }}$, the average growth rates of the three methods are $1.581 \%, 1.089 \%$, and $0.744 \%$, respectively; When $v_{\text {avg }}$ boosts, the average growth rates of the three methods are $1.803 \%, 1.427 \%$, and $1.082 \%$, respectively; with the increase in $\beta$, the average growth rates of the three methods are $0.441 \%, 0.401 \%$, and $0.211 \%$, respectively. We can effortlessly note that the increase of model parameters has the greatest impact on TUM and the least impact on ITUM. By comparison, we can observe that the $\beta$ is not the critical factor in the three parameters. The update operation of TUM is easily influenced by the number of fixed nodes within the sensing area and movement path of mobile node. TURIR triggers an update once the displacement of mobile node is higher than the fixed threshold value, and the displacement of mobile node is mainly ascertained by $v_{\text {avg }}$ and $d_{\text {avg }}$, as presented in Figures 7(a) and 7(b). The trigger updating mode of ITUM can adjust to the movement path and circumvent unnecessary updates within high similarity messages according to the adaptive dynamic threshold. Moreover, due to the anisotropic sensing model of ITUM, the sensing area of nodes is susceptible to the influence of $d_{\text {avg }}$ in mobility model, as shown in Figure 7(a).

From Figure 8, we can find that TUM and TURIR are both little affected by changes in information exchange rate $H$, as a result of the influence of timing updating mechanism and fixed threshold updating pattern, respectively. Specifically, the timing update mechanism applied by TUM has nothing to do with $H$, and the fixed threshold mode of TURIR is independent of $H$. The variation coefficients of the two methods are $0.308 \%$ and $0.415 \%$, respectively. The number of information exchanges of ITUM is positively correlated with $H$, and its variation coefficient is $41.475 \%$. It is 


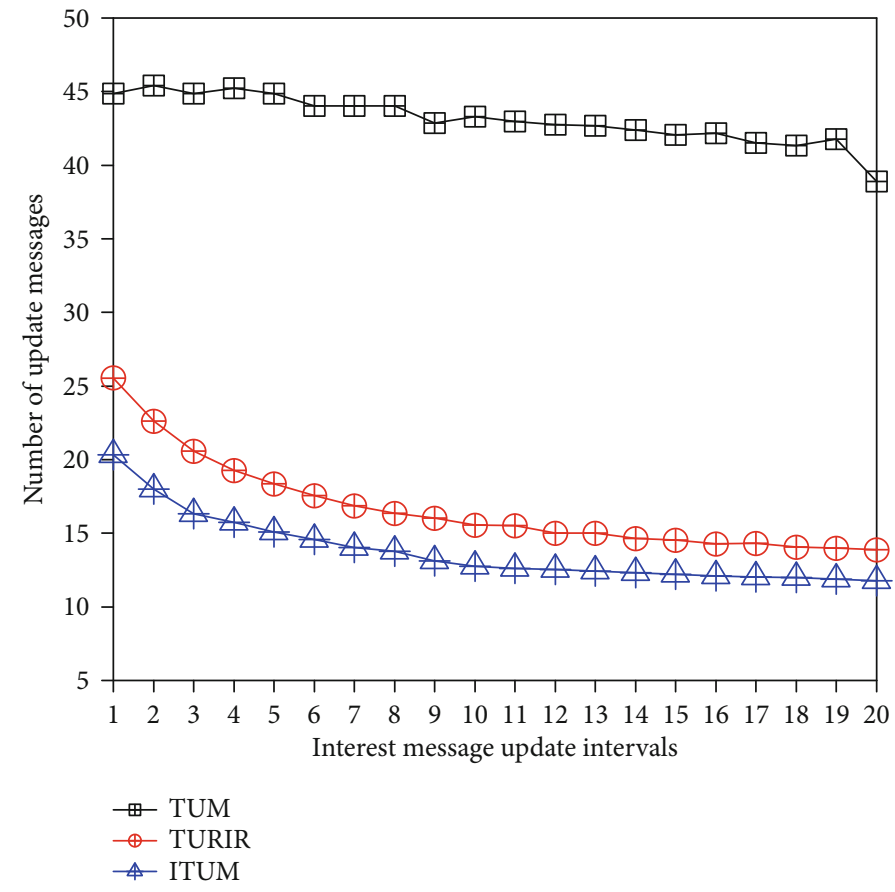

(a) Number of messages

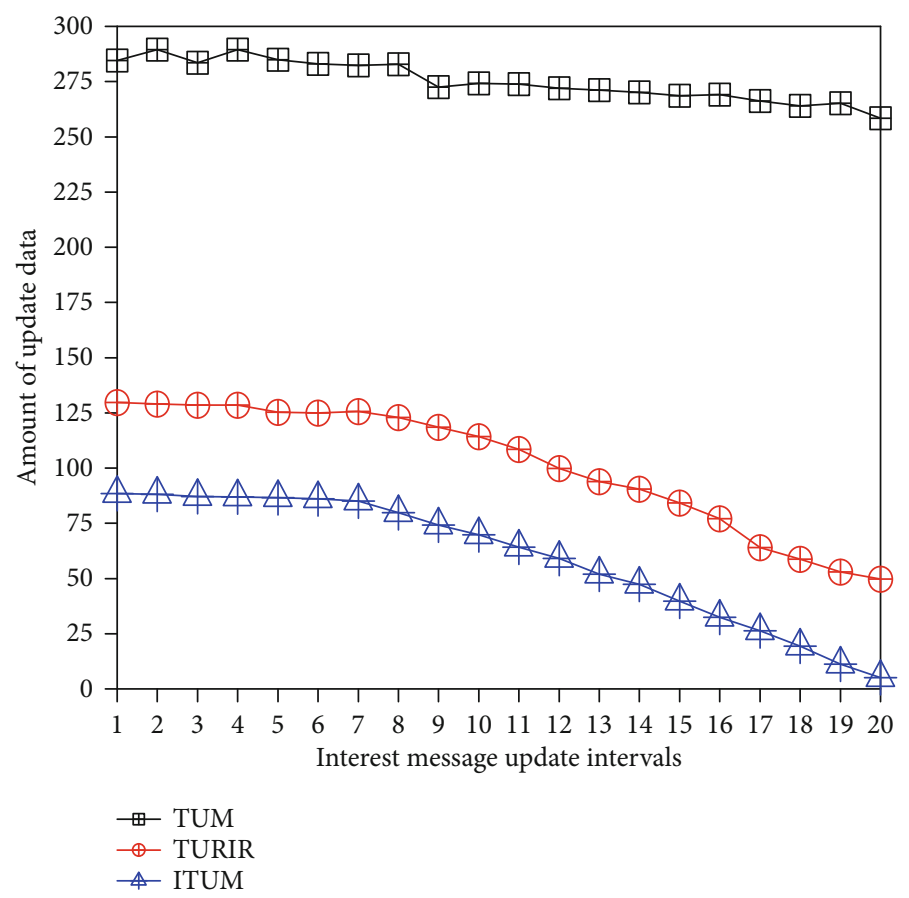

(b) Number of data

FigURE 6: Number of update messages and data of three methods varying interest message update interval.

because the probability of triggering updating increases with the growth of $H$, resulting in the increment of number of information exchanges.

In Figure 9, we can note that $m, H$, and $R$ have strong positive correlation with the number of information exchanges $(p<0.05)$. Although $L, v_{\text {avg, }}$ and $d_{\text {avg }}$ are also positively correlated with the number of information exchanges, the correlation is weaker compared with $m, H$, and $R$. $\beta$ has no concern with the number of information exchanges.

\section{Conclusion}

Energy efficiency is an essential concern in WSNs based on directed diffusion routing protocols. In this paper, after 


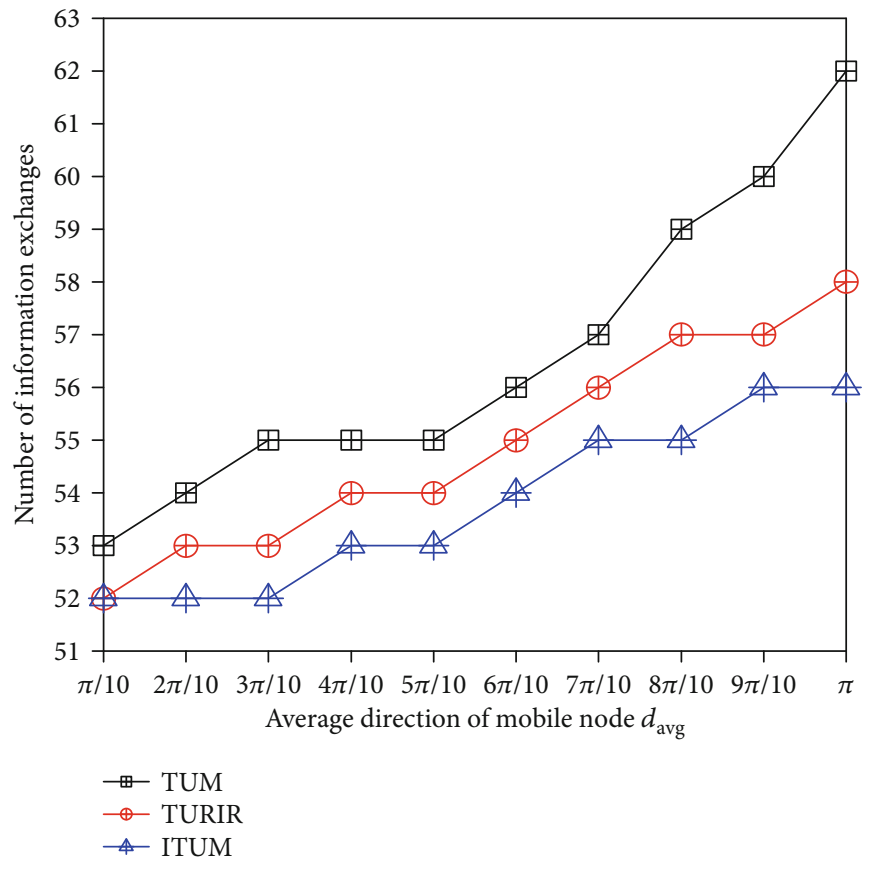

(a) Parameter $d_{\text {avg }}$

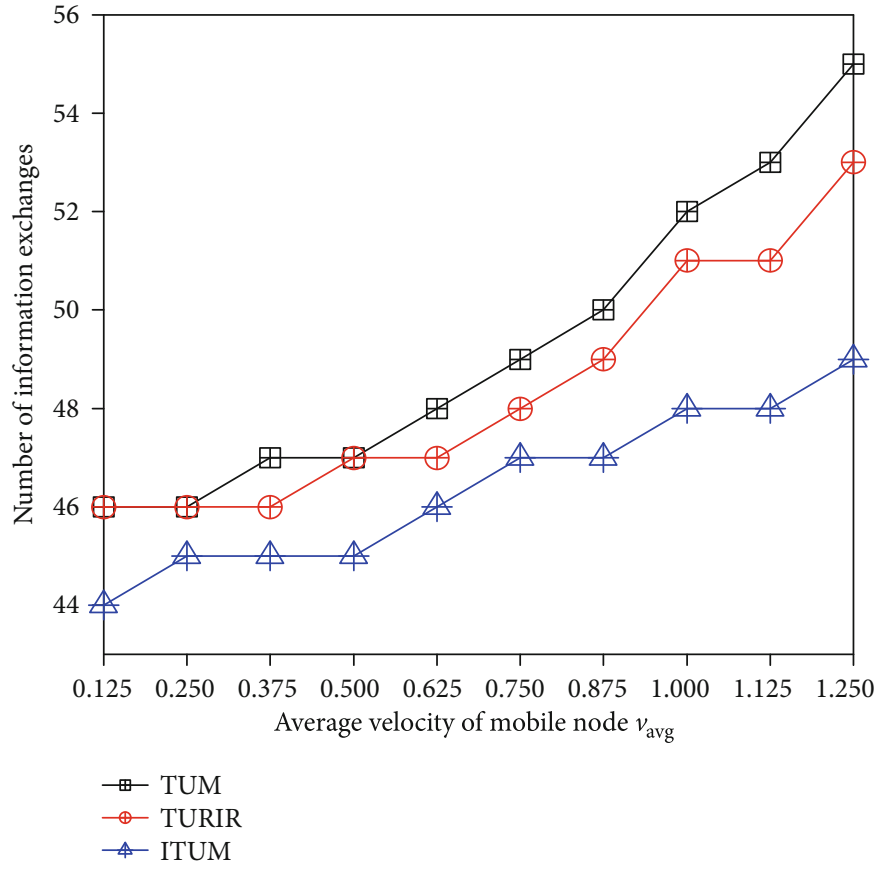

(b) Parameter $v_{\text {avg }}$

Figure 7: Continued. 


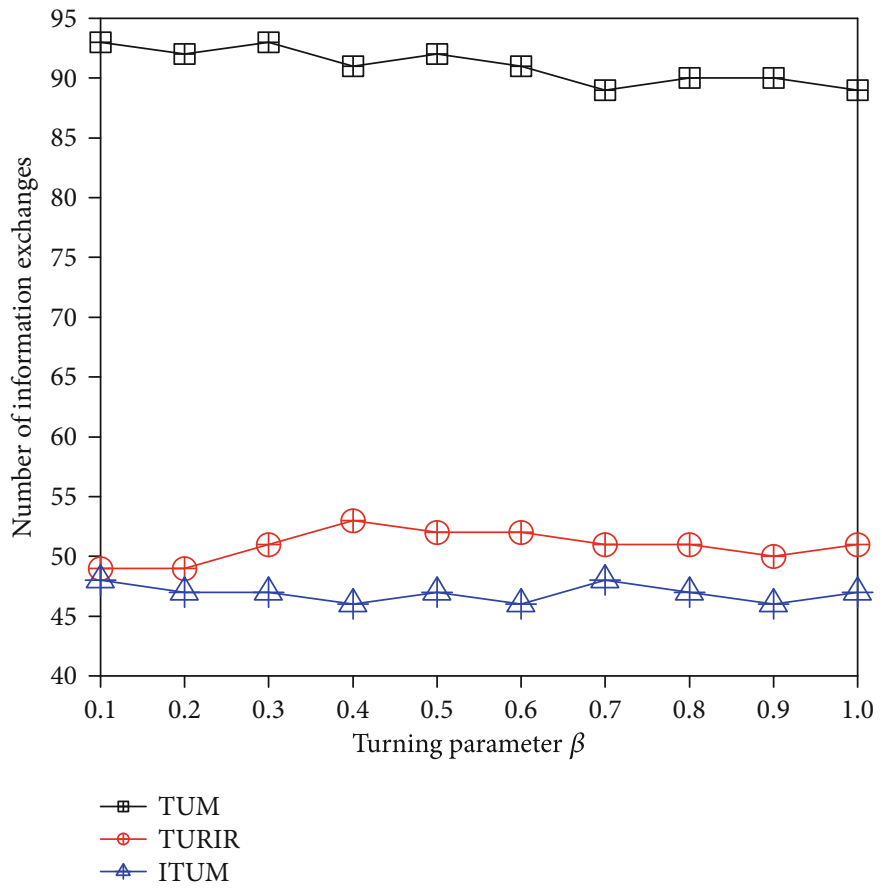

(c) Parameter $\beta$

Figure 7: Number of information exchanges of three methods varying movement model parameters.

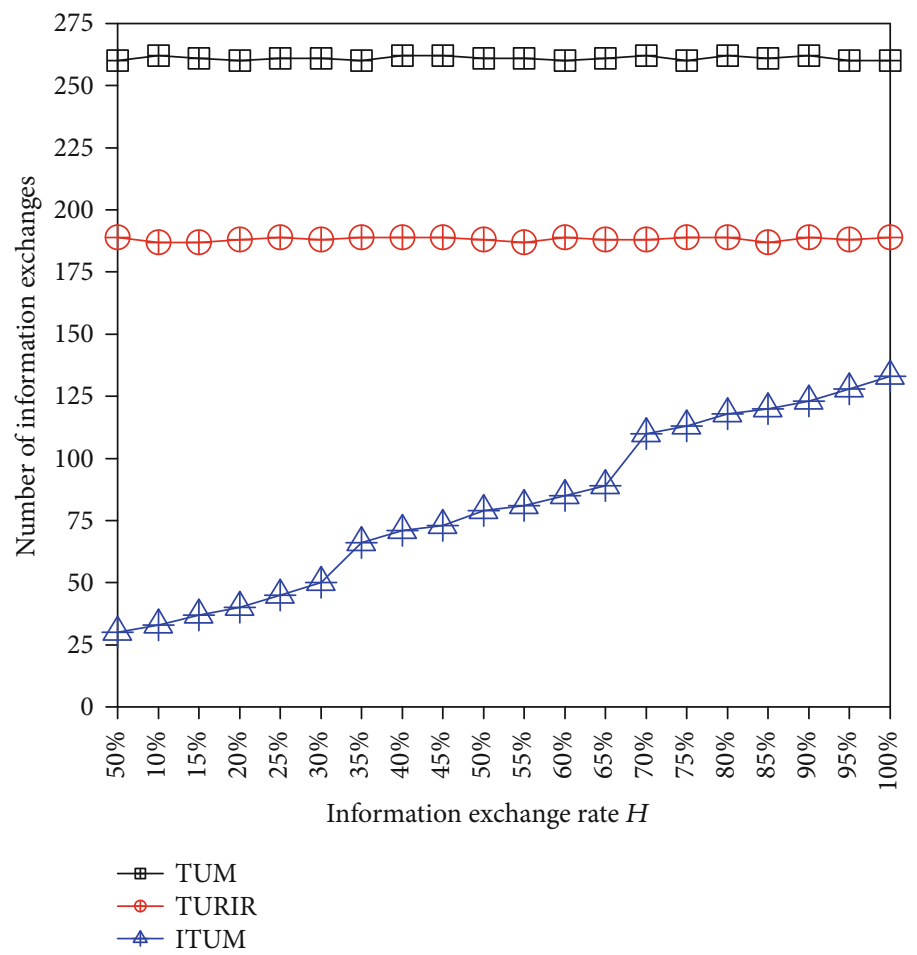

Figure 8: Relation between $H$ and number of information exchanges in different methods.

defining the network model and the sensing model, we established an improved triggering updating method of interest message to reduce the number of information exchanges through threshold determination based on AHP and update judgement using similarity calculation. The most significant advantage of this method is that it can better adapt the requirement of information exchange rate, according to the triggering updating mechanism composed of dynamic hierarchical threshold adjustment and message similarity comparison. Besides, we analyzed the impact of key parameters 


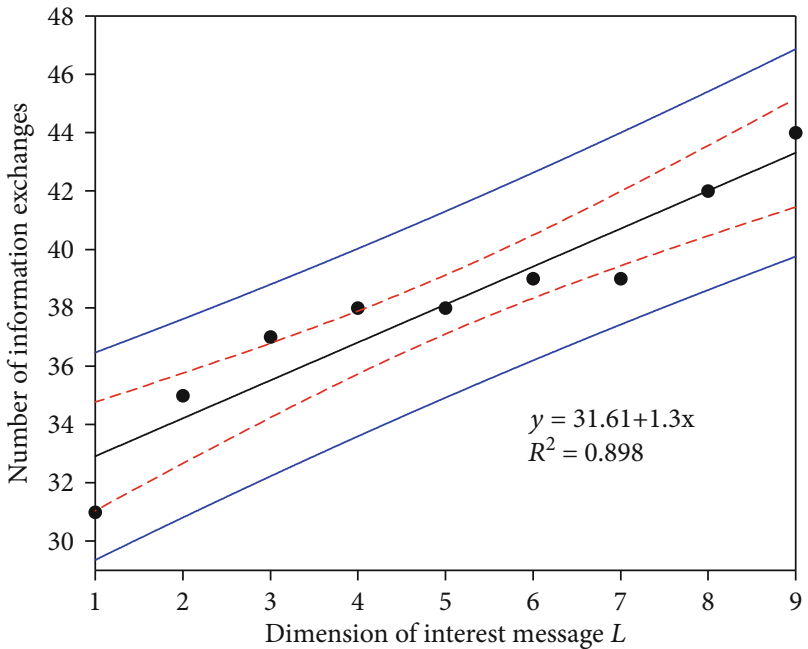

- Subject Fit curve

- - 95\% confidence interval - $95 \%$ forecast interval

(a)

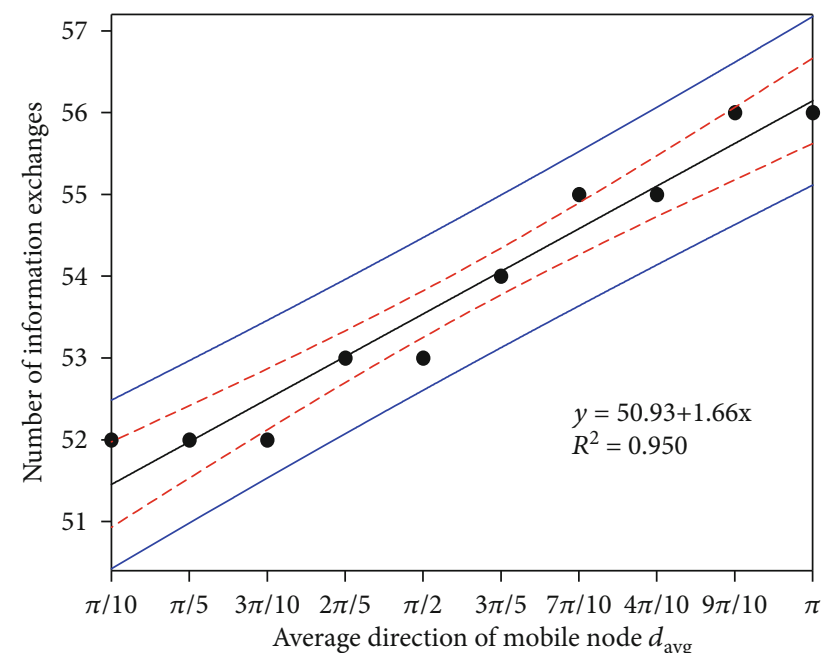

- Subject Fit curve

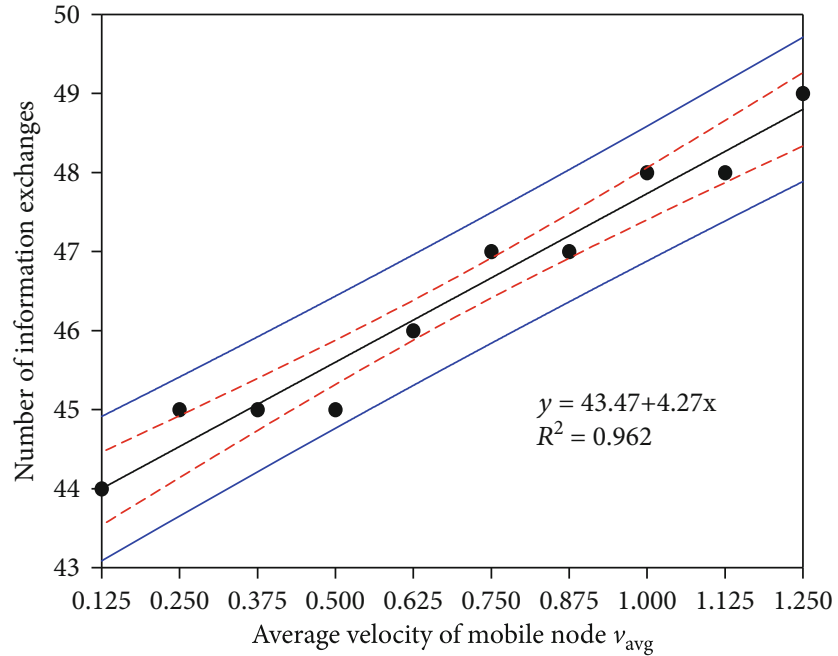

- Subject

Fit curve

- - - 95\% confidence interval — $95 \%$ forecast interval

(b)

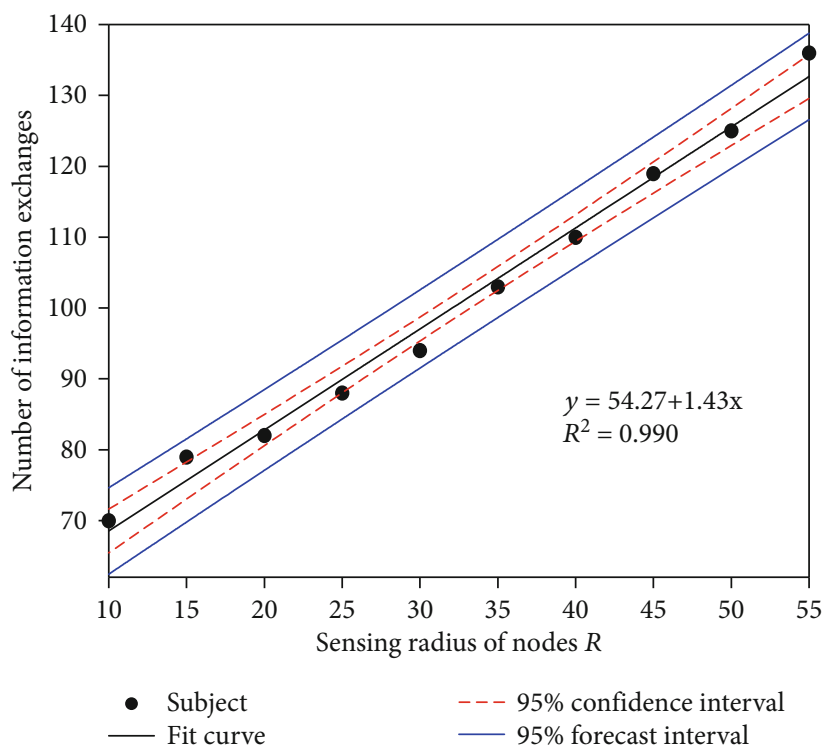

(d)

(c)

FIgure 9: Continued. 


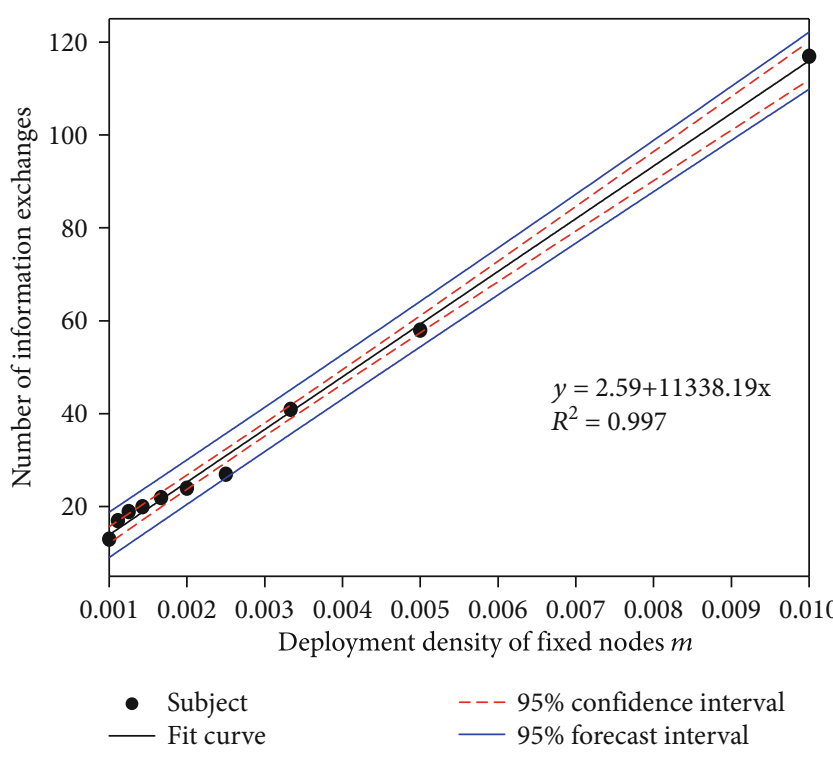

(e)

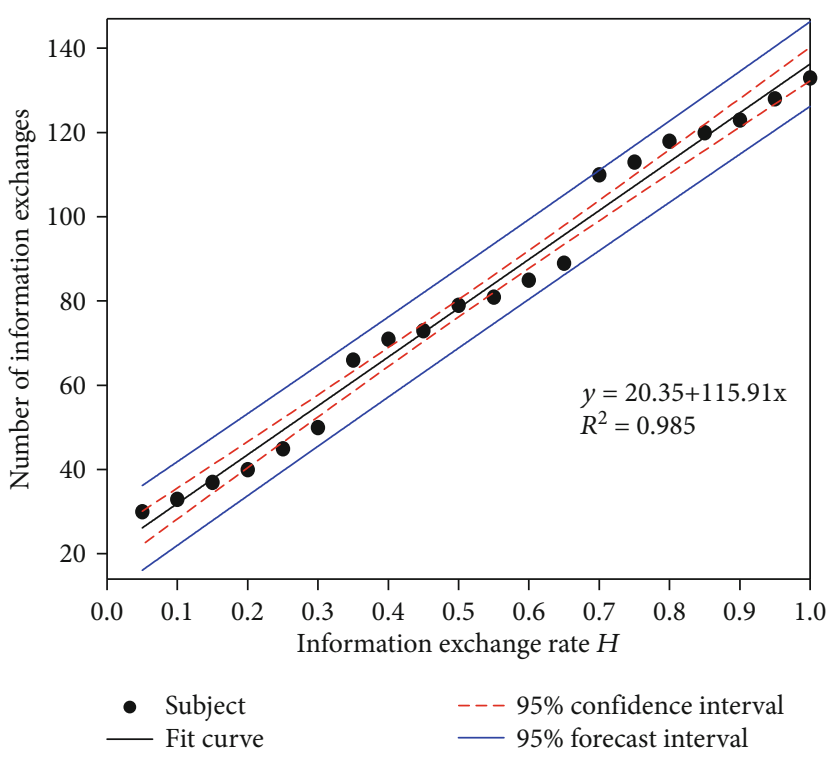

(f)

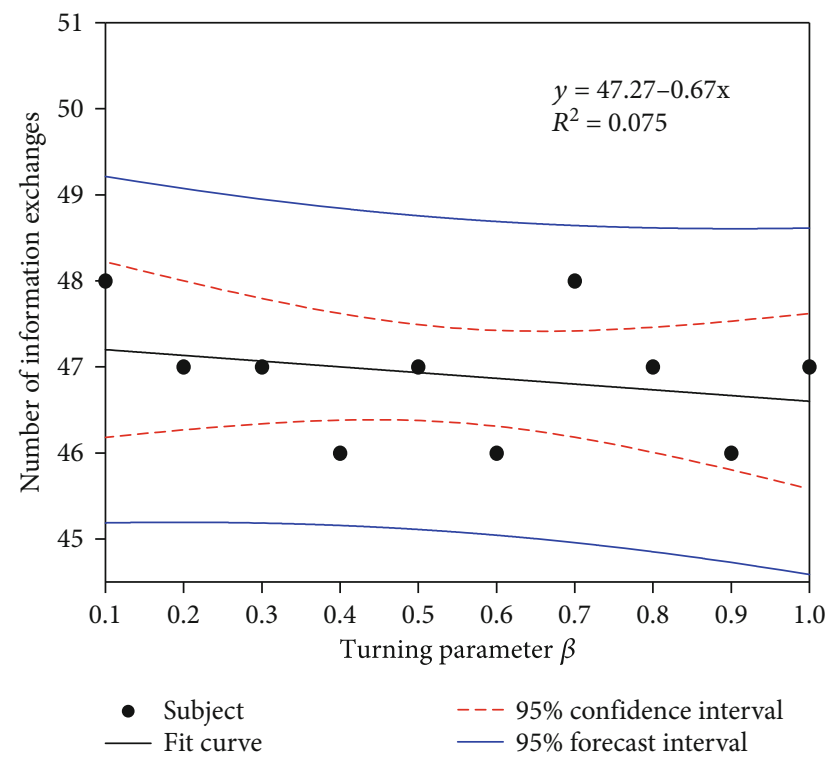

(g)

FIGURE 9: Linear correlation analysis of number of information exchanges and its influencing factors. (a-g) Represent linear relations between $L, v_{\mathrm{avg}}, d_{\mathrm{avg}}, R, m, H$, and $\beta$, respectively, and number of information exchanges.

and evaluated the performances of reducing update times with two interest message updating methods. The simulation results show that ITUM has a distinct advantage compared with the exiting interest message updating methods, and the proposed method can improve the adaptability to scene changes while lowering the number of information exchanges. Finally, other network constraints in real scenes, such as channel noise and packet loss, are not considered. It is this need in the next work to further test and improve the proposed method. Also, the paper provided the weight calculation method in threshold determination scheme is relatively complicated; in future works, the weight-decision method should be investigated to automatically get the recommended settings under the requirements imposed by a specific range of information exchange rates.

\section{Data Availability}

The authors declare that the data used to support the findings of this study are available from the corresponding author upon request.

\section{Conflicts of Interest}

The authors declared that they have no conflicts of interest to this work.

\section{Acknowledgments}

This work is supported by the National Natural Science Foundation of China (Grant no. 61771184), Program for 
Science \& Technology Innovation Talents in University of Henan Province (Grant no. 20HASTIT029), Program for Innovative Research Team (in Science and Technology) in University of Henan Province (Grant no. 19IRTSTHN021), and Science and Technology Major Project of Henan Province (Grant no. 181100110100).

\section{References}

[1] R. Kolcun, D. E. Boyle, and J. A. McCann, "Efficient distributed query processing," IEEE Transactions on Automation Science and Engineering, vol. 13, no. 3, pp. 1-17, 2016.

[2] S. G. El-Esawy, N. M. Elshennawy, and N. A. Elfishawy, "An improved energy-efficient directed diffusion routing protocol for wireless sensor network," in 2018 14th International Computer Engineering Conference (ICENCO), pp. 64-67, Cairo, Egypt, Egypt, December 2018.

[3] M. M. Khaing and T. M. Naing, "Energy aware data-centric routing in wireless sensor network," in International Conference on Advances in Engineering and Technology (ICAET'2014), pp. 80-83, Singapore, March 2014.

[4] S. Kaushal, N. Gupta, and R. Sandhu, "Comparative study of flooded and directed diffusion protocol in WSN," International Journal of Advanced Research in Electronics and Communication Engineering, vol. 5, no. 5, pp. 1213-1218, 2016.

[5] B. M. Mohammad el-Basioni, S. M. Abd el-kader, and H. S. Eissa, "Designing a local path repair algorithm for directed diffusion protocol," Egyptian Informatics Journal, vol. 13, no. 3, pp. 155-169, 2012.

[6] Y. Miao and Z. Tan, "Directed diffusion routing protocol based on cluster-head path transformation in wireless sensor network," Journal of Jilin University (Science Edition), vol. 55, no. 4, pp. 957-963, 2017.

[7] M. Omar, S. Yahiaoui, and A. Bouabdallah, "Reliable and energy aware query-driven routing protocol for wireless sensor networks," Annals of Telecommunications, vol. 71, no. 1-2, pp. 73-85, 2016.

[8] Y. Cui and H. Qin, "A novel rumor routing for wireless sensor network," in 2010 Fourth International Conference on Genetic and Evolutionary Computing, pp. 795-797, Shenzhen, China, December, 2010.

[9] J. N. Al-Karaki and A. E. Kamal, "Routing techniques in wireless sensor networks: a survey," IEEE Wireless Communications, vol. 11, no. 6, pp. 6-28, 2004.

[10] L. García Villalba, A. Sandoval Orozco, A. Triviño Cabrera, and C. Barenco Abbas, "Routing protocols in wireless sensor networks," Sensors, vol. 9, no. 11, pp. 8399-8421, 2009.

[11] S. Li, S. Duan, F. Li, and A. Liu, "An effective event query access strategy based on WSN," Journal of Central South University (Science and Technology), vol. 45, no. 7, pp. 2223-2230, 2014.

[12] K. P. K. Rao and K. Kalaiarasi, "Data-centric routing protocols in wireless sensor networks: a survey," European Journal of Advances in Engineering and Technology, vol. 2, no. 6, pp. 62-69, 2015.

[13] K. A. Rasbi, H. Shaker, and Z. Sharef, "Survey on data-centric based routing protocols for wireless sensor networks," International Journal of Electrical, Electronics and Computers, vol. 2, no. 2, pp. 9-16, 2017.

[14] C. Intanagonwiwat, R. Govindan, D. Estrin, F. Silva, and F. Silva, "Directed diffusion for wireless sensor networking,"
IEEE/ACM Transactions on Networking, vol. 11, no. 1, pp. 216, 2003.

[15] J. Mu, X. Yi, X. Liu, and L. Han, "An efficient and reliable directed diffusion routing protocol in wireless body area networks," IEEE Access, vol. 7, pp. 58883-58892, 2019.

[16] U. Rawat and M. Sharma, "Directed diffusion: features, current developments, issues and analysis," International Journal of Computer Applications, vol. 50, no. 12, pp. 31-35, 2012.

[17] Y.Xu, Y. Yang, Y. Xin, and H. Zhu, “An improved directed diffusion protocol based on opportunistic routing," Journal of Networks, vol. 9, no. 5, pp. 1163-1168, 2014.

[18] M. Chen, T. Kwon, and Y. Choi, "Energy-efficient differentiated directed diffusion (EDDD) in wireless sensor networks," Computer Communications, vol. 29, no. 2, pp. 231-245, 2006.

[19] K. E. Kannammal and T. Purusothaman, "Performance of improved directed diffusion protocol for sensor networks under different mobility models," Journal of Computer Science, vol. 8, no. 5, pp. 694-700, 2012.

[20] A. N. Eghbali and M. Dehghan, "Load-balancing using multipath directed diffusion in wireless sensor networks," Mobile Ad-Hoc and Sensor Networks. MSN 2007, H. Zhang, S. Olariu, J. Cao, and D. B. Johnson, Eds., , pp. 44-55, Springer, Berlin, Heidelberg, Berlin, German, 2007.

[21] Z. Li and H. Shi, "Design of gradient and node remaining energy constrained directed diffusion routing for WSN," in 2007 International Conference on Wireless Communications, Networking and Mobile Computing, pp. 2600-2603, Shanghai, China, September 2007.

[22] X. Liu, F. Li, H. Kuang, and X. Wu, "The study of directed diffusion routing protocol based on clustering for wireless sensor network," in 2006 6th World Congress on Intelligent Control and Automation, pp. 5120-5124, Dalian, China, June 2006.

[23] F. Semchedine, L. Bouallouche-Medjkoune, M. Tamert, F. Mahfoud, and D. Aïssani, "Load balancing mechanism for data-centric routing in wireless sensor networks," Computers and Electrical Engineering, vol. 41, pp. 395-406, 2015.

[24] N. S. Samaras and F. S. Triantari, "On direct diffusion routing for wireless sensor networks," in 2016 Advances in Wireless and Optical Communications (RTUWO), pp. 89-94, Riga, Latvia, November 2016.

[25] A. Merlo, M. Migliardi, D. Raso, and E. Spadacini, "Optimizing network energy consumption through intrusion prevention systems," in International Joint Conference SOCO'14CISIS'14-ICEUTE'14, pp. 505-515, Bilbao, Spain, June 2014.

[26] N. Mehta and N. V. Doohan, “An energy efficient approach for highly data-centric directed diffusion in wireless sensor networks," International Journal of Electrical, Electronics and Computer Engineering, vol. 1, no. 1, pp. 11-14, 2012.

[27] J. Yu and H. Zhang, "Directed diffusion based on clustering and inquiry for wireless sensor networks," in 20103 rd International Conference on Computer Science and Information Technology, pp. 291-294, Chengdu, China, July 2010.

[28] Y. Cui and J. Cao, "An improved directed diffusion for wireless sensor networks," in 2007 International Conference on Wireless Communications, Networking and Mobile Computing, pp. 2380-2383, Shanghai, China, September 2007.

[29] B. Krishnamachari and J. Ahn, "Optimizing data replication for expanding ring-based queries in wireless sensor networks," in 2006 4th International Symposium on Modeling and Optimization in Mobile, Ad Hoc and Wireless Networks, pp. 1-10, Boston, USA, March 2006. 
[30] L. Niu, L. Liu, and Q. Xia, "TURIR: energy efficient interest message update protocol for mobile wireless sensor networks," Chinese Journal of Sensors \& Actuators, vol. 22, no. 4, pp. 511519, 2009.

[31] G. Zhou, T. He, S. Krishnamurthy, and J. A. Stankovic, "Models and solutions for radio irregularity in wireless sensor networks," ACM Transactions on Sensor Networks, vol. 2, no. 2, pp. 221-262, 2006.

[32] H. Saito, "Theoretical optimization of sensing area shape for target detection, barrier coverage, and path coverage," IEICE Transactions on Communications, vol. E99.B, no. 9, pp. 1967-1979, 2016.

[33] A. Wei, F. Shao, and H. Meng, "The coverage-control optimization in sensor network subject to sensing area," Computers and Mathematics with Applications, vol. 57, no. 4, pp. 529539, 2009.

[34] T. L. Saaty, "How to make a decision: the analytic hierarchy process," European Journal of Operational Research, vol. 48, no. 1, pp. 9-26, 1990.

[35] M. T. Mastura, S. M. Sapuan, and M. Noryani, "Material selection of natural fibers for composite automotive component using analytic hierarchy process/analytic network process in concurrent engineering approach," Key Engineering Materials, vol. 801, pp. 53-58, 2019.

[36] R. M. Czekster, H. J. De Carvalho, G. Z. Kessler, L. M. Kipper, and T. Webber, "Decisor: a software tool to drive complex decisions with analytic hierarchy process," International Journal of Information Technology and Decision Making, vol. 18, no. 1, pp. 65-86, 2019.

[37] J. Yuan and X. Liang, "Consistency improvement of judgment matrix in analytic hierarchy process," Statistics \& Decisions, vol. 30, no. 12, pp. 15-17, 2014.

[38] S. Huang and Q. Chen, "On clustering algorithm of high dimensional data based on similarity measurement," Computer application and software, vol. 26, no. 9, pp. 102-105, 2009. 